MS15-04

\section{Natural phosphides as indicators of planetary systems evolution}

Sergey Britvin ${ }^{1}$, Michail Murashko², Maria Krzhizhanovskaya ${ }^{1}$, Yevgeny Vapnik ${ }^{3}$, Sergey Krivovichev ${ }^{4}$

1. Department of Crystallography, Institute of Earth Sciences, SaintPetersburg State University, Saint-Petersburg, Russia

2. Saint-Petersburg State University, Saint-Petersburg, Russia

3. Ben-Gurion University of the Negev, Beer-Sheva, Israel

4. Kola Science Center, Russian Academy of Sciences, Apatity, Murmansk Region, Russia

email: sbritvin@gmail.com

Natural phosphides is a specific class of minerals containing phosphorus in a redox state lower than zero. The occurrence of phosphide minerals always indicate on highly reducing geo- or cosmochemical environment. At the same time, phase transformations of phosphides are the sensitive indicators of $P-T$ conditions occurred during planetary systems formation. ${ }^{1,2}$ Phosphides are the common constituents of meteorites; however, till now they were virtually unknown on the Earth. Our recent findings demonstrate that phosphide minerals could play an essential role at the early stages of the Earth's history, in Archean era. ${ }^{3}$ We herein summarize the results of the recent studies of natural phosphides, with the emphasis on cosmochemically important system $\mathrm{Fe}-\mathrm{Ni}-\mathrm{P}$, and provide the insights on the role of these minerals in planetary systems evolution.

This study was carried out under financial support of Russian Scientific Foundation, grant no. 18-17-00079. The authors thank Center for X-ray diffraction studeies of Saint-Petersburg State University for instrumental and computational support.

References:

[1] Britvin, S. N., Rudashevsky, N. S., Krivovichev, S. V., Burns,

P. C. \& Polekhovsky, Y. S. (2002). American Mineralogist, 87,

$1245-1249$.

[2] Dera, P., Lavina, B., Borkowski, L. A., Prakapenka, V. B., Sutton, S. R., Rivers, M. L., Downs, R. T., Boctor, N. Z. \& Prewitt, C. T. (2008). Geophysical Research Letters, 35, L10301.

[3] Britvin, S. N., Murasko, M. N., Vapnik, Ye., Polekhovsky, Yu. S. \& Krivovichev, S. V. (2015). Scientific Reports, 5, 8355.

Keywords: phosphide, planetary system, mineral

\section{MS15-05}

\section{In-situ studies of clathrate hydrates on icy solar system bodies}

Sarah Day ${ }^{1}$, Emmal Saf ${ }^{2}$, Stephen P Thompson ${ }^{1}$, Aneurin Evans ${ }^{3}$, Claire Murray $^{1}$, Julia Parker ${ }^{1}$

1. Diamond Light Source, Didcot, United Kingdom

2. Newcastle University, Newcastle, United Kingdom

3. Keele University, Newcastle-Under-Lyme, United Kingdom

email: sarah.day@diamond.ac.uk

Clathrate hydrates are compound structures consisting of water ice, in which water molecules form cage structures capable of trapping a variety of gas molecules. There is considerable evidence to suggest that clathrate hydrates are present on a number of bodies in the Solar System, playing important roles in their atmospheric evolution and surface processes. For example, the origin of the energetic plumes seen emanating from the south pole of Saturn's moon, Enceladus, by Cassini has been associated with the dissociation of large quantities of clathrates hydrates [1]. This is due to the fact that the plumes have been found to contain gases such as $\mathrm{CH}_{4}$ and $\mathrm{N}_{2}$, both of which have poor solubility in liquid water On Mars, clathrates have been postulated to play an important role in the distribution and total inventory of Martian volatiles, including methane and noble gases (Xe, Ar, Kr), and that the dissociation of extensive sub-surface clathrate deposits could be responsible for some of the geomorphological features observed on the surface of the planet [2]. They have similarly been suggested as a mode of depletion for the noble gases in the atmosphere of Titan and as the source of the nitrogen depletion observed in comets. Additionally, clathrates are also of terrestrial importance, being recognised as potentially important materials for future energy recovery and storage, as well as $\mathrm{CO}_{2}$ sequestration. Clathrates are, however, notoriously unstable, existing only in specific temperature and pressure regimes that depend on the size of the trapped gas molecules, and the structure of the ice cages themselves. While a number of thermodynamic models exist to describe their behaviour, experimental data to support these are lacking, especially in the case of real world systems in which clathrates are forming in environments other than pure water, such as saline oceans.

In order to improve our understanding of the structural properties of clathrates and their contribution to the shaping and evolution of these bodies we have conducted in-situ synchrotron X-Ray powder diffraction measurements, in conjunction with Raman spectroscopy and small-angle scattering, at Diamond Light Source, UK. The properties of $\mathrm{CO}_{2}$ and $\mathrm{CH}_{4}$ clathrates forming in a variety of saline solutions, over a range of temperature and pressure conditions, analogous to those found on Enceladus, Europa, Mars and Titan have been studied. Details of the experimental setup used and the results of these studies will be presented here.

References:

[1] Kieffer, S. W., Lu, X., Bethke, C.M. et al. (2006). Science, 314,

1764-1766

[2] Mousis, O., Chassefière, E., Lasue, J., et al. (2013). Space

Science Reviews, 174, 213-250

Keywords: Clathrates, X-Ray Diffraction, Solar System 